\title{
Residual Network Structure-based High Accuracy Spectral Analysis Method
}

\author{
Sai Wu ${ }^{a}$,* Zhihui Wang ${ }^{\mathrm{a}}$, Sachura Meng ${ }^{\mathrm{a}}$, Weijun Zheng ${ }^{\mathrm{b}}$, and Weiping Shao ${ }^{\mathrm{c}}$ \\ ${ }^{a}$ China Electric Power Research Institute, Beijing, 100192, China \\ ${ }^{b}$ State Grid Jiaxing Power Supply Company, Jiaxing, 314000, China \\ ${ }^{c}$ State Grid Zhejiang Electric Power Limited Company, Hangzhou, 310000, China
}

\begin{abstract}
The pivotal technology in spectrum analysis is the classification for normal communication signals and interference ones. Automatic modulation classification (AMC) is widely utilized to identify modulation types of received signals. In this paper, original signals with different modulation types are taken as the original input of the network. A convolutional neural network with residual network structure is designed to identify the modulation type. Meanwhile, a sliding window method is proposed to expand the data set. RadioML2016.a data sets are utilized for simulation, and the simulation results indicate that the complexity and accuracy of this method are better than those of recent methods.
\end{abstract}

Keywords: automatic modulation classification; sliding window; residual network

(Submitted on September 30, 2019; Revised on November 2, 2019; Accepted on November 12, 2019)

(C) 2019 Totem Publisher, Inc. All rights reserved.

\section{Introduction}

The key technology of spectrum analysis is the quick recognition for normal communication signals and the interference ones. In order to achieve the above purpose, quick identification of the modulation type of the input signal is needed. Automatic modulation classification (AMC), which is viewed as a key step between the signal detection and demodulation, can automatically identify the type of received signal from the received data samples. AMC plays an important role in a variety of cooperative and non-cooperative communication applications, such as software-defined radio, cognitive radio, smart modems, surveillance, and electronic warfare [1].

AMC is mainly divided into likelihood-based and feature-based methods [2-4]. The likelihood-based method considers $\mathrm{AMC}$ as a hypothesis testing problem and can obtain high classification accuracy by minimizing the probability of misclassification when both channel models and noises are given, but it is not widely used in practical operations due to its high computational complexity and low robustness. In contrast, although the feature-based method extracts a series of statistical features from the received signals, it achieves suboptimal classification performance compared with LB methods. FB methods have the advantages of low computational complexity, high efficiency, and better robustness to model mismatches. Hence, feature-based AMC methods are often used in practice, as no more parameter estimation is needed to assist the AMC process. Moreover, it is noted that we can improve the classification performance of FB method by increasing the length of the received signal symbols.

Among feature-based recognition methods, artificially extracted statistical features such as high-order cumulant features [5-6], high-order cyclic cumulant features [7] and cyclic spectral features [8] are often utilized. Gardner et al. developed a cyclostationary-based modulation classification method, which can achieve superior performance in a low signal-to-noise (SNR) environment by using cyclic spectrum features [9]. However, the high computational complexity and the knowledge of the symbol rate of the received signal limit the application of this method. Kim et al. constructed a low complexity cyclic domain feature using the maximum of cyclostationary spectrum over frequency [10]. However,

* Corresponding author.

E-mail address:wusai2008@163.com 
cyclostationary-based AMC only can deal with the classification problem of M-ary frequency shift keying (MFSK) and M-ary amplitude shift keying (MASK), and it is unable to deal with the classification problem of M-ary phase shift keying (M-PSK) and M-ary quadrature amplitude modulation (M-QAM). Swami et al. proposed a cumulant-based hierarchical modulation classification framework to conduct modulation classification. The robustness to phase offset was achieved and verified, but the decision was made using the minimum distance criterion, which led to sub-optimal classification performance [11]. Moreover, since different features have different characteristics, manual feature selection and decision thresholds should be conducted on the experience, and this may largely influence the classification performance. Therefore, this paper focuses on improving the classification accuracy of FB methods and avoiding experience-based manual feature selection.

Machine learning theory has often been used in automatic modulation classification in recent years because of its unified framework and high characterization [12-14]. On this base, the received signal can be well represented, and manual feature selection and threshold settings can be avoided. Narendar [15] proposed a support vector machine (SVM)-based signal recognition method, which uses high-order cumulant as the original input feature and SVM to make decisions. It compensates for the performance loss of feature-based recognition methods by increasing the number of samples. However, the features extracted by artificial design are not optimal for the characterization of the original signal. The ideal algorithm should have the ability to automatically learn the features more than manually extracted ones.

The deep learning-based method combines various neural network structures to automatically learn and extract new features from the original input according to the target [16-17]. Thus, the features obtained are highly characterized and well suitable for the following network. The SCAE (stacked convolutional auto-encoders) method firstly transforms high-order cumulant features into feature matrices and then uses SCAE for unsupervised automatic encoding and decoding in the offline training process. It makes the classification decisions in the same process in the on-line classification process [18]. This method can automatically extract features and learn classification, but the computational complexity is high and the processing of the original signal is complicated. In order to reduce the complexity of the original features, [19] proposed a method based on RNN (recurrent neural network) using LSTM (long-short term memory) structure. The amplitude-phase information of the signal was taken as the original input, and the classification was performed using classifiers that are good at processing sequence features. A high classification accuracy was obtained, but the computational complexity was high.

In this paper, an automatic modulation recognition method named AP-ResNet, which is based on CNN (convolutional neural network) with ResNet (residual network) structure, is proposed for reducing the time complexity and achieving better classification accuracy. The method takes the amplitude-phase information of the signal as the original input, and a convolutional neural network with residual network structure is designed to identify the modulation type. Moreover, a sliding window method is proposed to expand the data set. RadioML2016.a data sets are utilized for simulation, and the simulation results indicate that the complexity and accuracy of this method are better than those of recent methods.

\section{Signal Model and Method}

\subsection{Signal Model}

Assume that the receiver is equipped with a single antenna and receives signals from one source. The received baseband signal can be shown as Equation (1).

$$
x(t)=s(t) \times c(t)+n(t)
$$

Where $s(t)$ is the envelope of the noiseless baseband complex signal and $n(t)$ denotes the additive white Gaussian noise. $c(t)$ is the time-varying impulse response of the wireless transmission channel, and we assume that it is invariant during the classification process. In addition, the term $s(t)$ is the complex signal symbol belonging to a predefined constellation with unit power.

The basic task for automatic modulation classification is to calculate the probability $P\left(s(t) \in N_{i} \mid r(t)\right)$ and select one as the decision making result according to the maximum likelihood criterion, where $x(t)$ represents the received signal and $N_{i}$ denotes the $i^{\text {th }}$ modulation class. Figure 1 presents the automatic modulation classification framework of this paper, which can be coarsely divided into two modules, i.e., a feature extraction module and an AP-ResNet-based classification module. Generally speaking, in the feature extraction module, the received signal $x(t)$ is expressed as the complex signal with the form of real part I and imaginary part $Q$, which is more flexible and simple in mathematical operation and can be obtained as 


$$
\begin{aligned}
& I=\operatorname{Re}(x(t)) \\
& Q=\operatorname{Im}(x(t))
\end{aligned}
$$

Where $\operatorname{Re}(x(t))$ and $\operatorname{Im}(x(t))$ denote the real and imaginary parts of the received signal, respectively. On this base, the amplitude and the phase feature of the received signal can be given by

$$
\begin{gathered}
A=\sqrt{I^{2}+Q^{2}} \\
P=\arctan (Q / I)
\end{gathered}
$$

Therefore, according to the input IQ information, the amplitude and phase feature of the signal can be calculated.



Figure 1. The diagram of the AMC framework

On the basis of the extracted amplitude and phase feature, we first train the proposed AP-ResNet network by using the amplitude and phase feature as the input. Secondly, in the classification process, the trained AP-ResNet network extracts high representative vectors and obtains a $\mathrm{K}$ score value, where $\mathrm{K}$ is the number of corresponding candidate modulation sets. By comparing the score values, the hypothesis with the largest score is determined as the final modulation type.

\subsection{Introduction of Data Set}

In this paper, the RadioML data set is utilized to assess the performance of the proposed model. The standard modulation data set shown in [16] is applied as a benchmark for training and evaluating model performance. This paper uses the RadioML 2016.10a data set, which is synthesized by GNU Radio [17] with commercial modulation parameters. The data set contains many real channel defects, such as phase frequency offset, sampling rate offset, additive white Gaussian noise, and multipath fading. Detailed specifications and generating details of the data can be found in [16].

\subsection{Residual Structure-based CNN}

In the field of deep learning, to improve the training efficiency and performance of the network, it is necessary to consider deepening the network layers. In recent years, the proposed residual network structure has increased the network efficiency in deep learning. In this paper, the amplitude A and phase $\emptyset$ information of the original signal are cut at a fixed length as original input. At the same time, a residual network structure based deep learning method is designed to carry out automatic feature learning of the original input information. The overall structure of the method is shown in Figure 2. The original amplitude and phase sequence pass through six residual stacks, then through the fully-connected layer and the soft-max layer. Finally, the output features are matched into the five categories, which respectively represent five types of modulation modes.

The internal structure of the residual stack is shown in Figure 3. Firstly, the input passes through a convolutional kernel with the size $1 \times 1$. Then, it enters into two residual units. Finally, it passes through the pooling layers with the size $1 \times 2$ and using step 2. The residual unit is the key point of the residual network classification structure, whose internal structure is shown in Figure 4. The concept of bypass connection is applied here. Compared with the traditional VGG (visual geometry group) structure, the residual unit based network structure can slow down overfitting and improve the classification accuracy. The size of convolutional layer in Figure 4 is $1 \times 3$, and the stride is 1 .

The parameter settings of the neural network in this paper are shown in Table 1. 


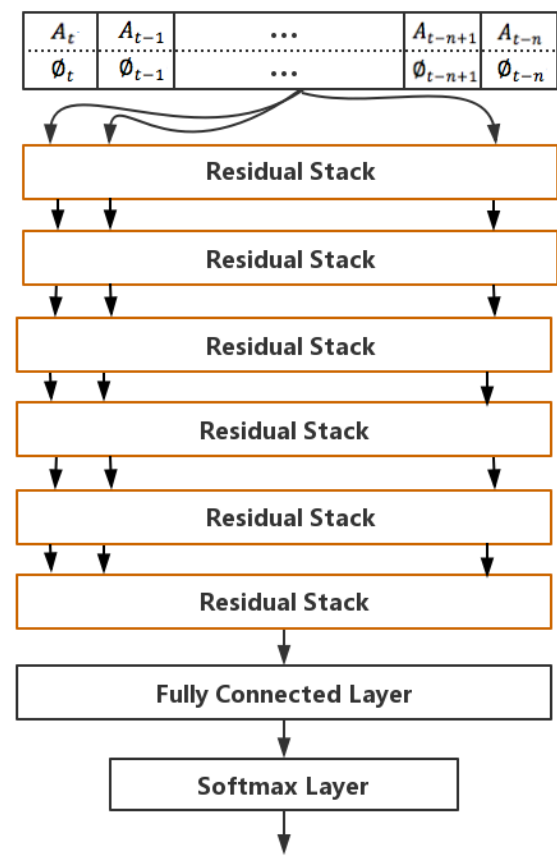

Figure 2. The diagram of the network



Figure 3. Internal structure of the residual stack

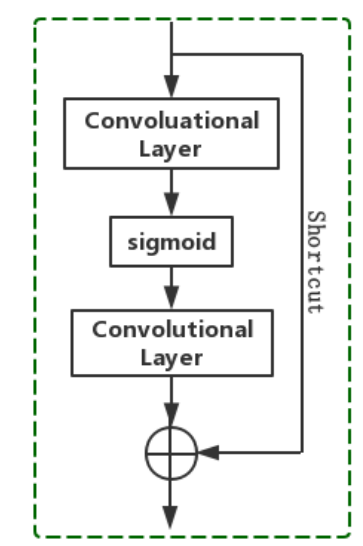

Figure 4. Internal structure of the residual unit

Table 1. Parameters setting of the residual network

\begin{tabular}{ccc}
\hline Layer & Input size & Activation function \\
\hline Input Layer & $2 \times n$ & ReLUs \\
Residual Stack & $32 \times n / 2$ & ReLUs \\
Residual Stack & $32 \times n / 4$ & ReLUs \\
Residual Stack & $32 \times n / 8$ & ReLUs \\
Residual Stack & $32 \times n / 16$ & ReLUs \\
Residual Stack & $32 \times n / 32$ & ReLUs \\
Residual Stack & $32 \times n / 64$ & ReLUs \\
Fully Connected Layer & 5 & Softmax \\
\hline
\end{tabular}

\subsection{Feature Engineering based on Sliding Window Method}

In actual applications, we often encounter the situation that the amount of the data set is small, leading to too few features extracted. Therefore, the original data set needs to be expanded. In the past, there were many expansion methods, such as 
duplicating samples repeatedly to form a large number of data and repeatedly splitting sample sets more than once randomly. Although these expansion methods expand the number of sample sets, they do not consider the time-domain correlation within sample sets. These methods may not work in expanding the classification features. Considering the intra-sample set correlation, in this paper, we propose an expansion method based on the sliding window method, which can not only retain the intra-sample correlation characteristics, but also expand the number of samples to achieve the purpose of expanding the data set. The expansion diagram is shown in Figure 5.



Assume the length of the original sample set is $n$. The length of the sliding window is set as $n / 2$, as shown by the red box. The sliding window slides along the direction shown by the arrow in the figure, intercepting one sample set with a length of $n / 2$ at every 1 sample unit, until the right end of the slide window coincides with the right end of the sample set. If $n / 2$ is taken as the unit sample, the original sample number is 2 . After using the sliding window method, the sample number increases to $n / 2$, and $(n / 2-2)$ is expanded. When $n>4$, the original sample data set is always expanded.

\section{Simulation Results}

In this section, extensive simulations are conducted to illustrate the performance of the convolutional neural network with residual network structure. The mobile communication system standards, such as LTE and 5G, are mostly PSK and QAM. The modulation formats between QAM and PSK in low SNR are difficult to discriminate. Therefore, we assume the candidate modulation formats are \{BPSK, QPSK, 8-PSK, 16-QAM, 64QAM\} with equal prior probability. For each predefined symbol length and SNR, ten thousand Monte Carlo trials are performed to obtain the probability of the correct classification. Each modulation is randomly divided into a training set and a test set according to the ratio of 4:1. Softmax is selected as the loss function, and the SNRs ranges from $-5 \mathrm{~dB}$ to $15 \mathrm{~dB}$ with an interval of $2 \mathrm{~dB}$. The training set size is [40 $000 \times 2 \times N]$, and the test set size is [10 $000 \times 2 \times N]$. Residual network parameters are set as shown in Table 1 . With the Adam optimizer, the learning rate is 0.001 , and the learning rate is reduced by $50 \%$ every 50 cycles.

\subsection{Classification Accuracy for Different Signal Lengths}

In Figure 6, we explore the classification performance of the proposed method versus SNRs with different symbol lengths. The SNR ranges from $-5 \mathrm{~dB}$ to $15 \mathrm{~dB}$. It is observed that the classification accuracy increased with more signal symbols and the gains of using 1024 symbols, 512 symbols, and 256 symbols over 128 symbols are $2.5 \mathrm{~dB}, 2 \mathrm{~dB}$, and $1 \mathrm{~dB}$, respectively, at $80 \%$ Pcc. Moreover, the classification accuracy of the proposed method could achieve $100 \%$ in $7 \mathrm{~dB}$, demonstrating its accuracy.

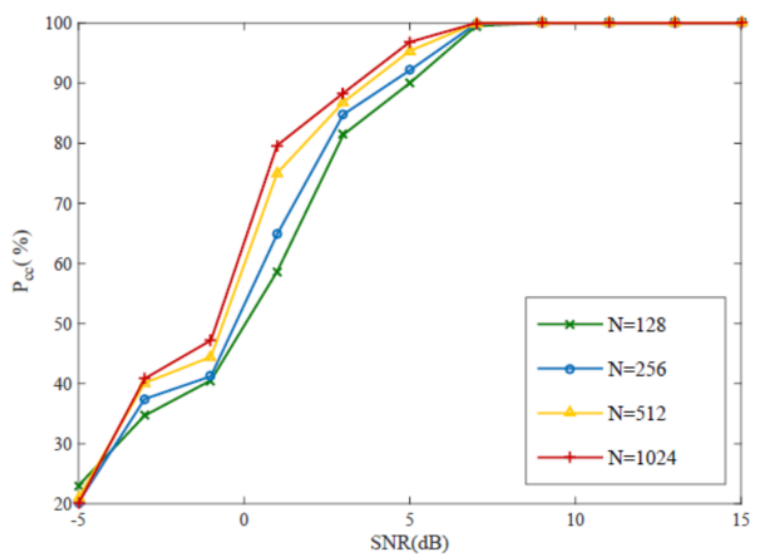

Figure 6. The classification accuracy of this method with different nodes 


\subsection{Classification Accuracy of Different Methods}

Figure 7 reveals the performance comparisons between AP-ResNet and the other methods, i.e., SVM, SCAE, and LSTM. Specifically, SVM is based on higher-order cumulant, and SCAE firstly converts the cumulant to matrix and then classifies by the convolutional autoencoder. The combination of RNN and LSTM, which involves gate-structure, solves the problem of gradient disappearance and gradient explosion. In Figure 7, it can be observed that the proposed AP-ResNet performs better than SVM [7], SCAE [8], and LSTM [15]. This can be explained from three aspects. Firstly, compared with APResNet, SVM depends on artificial features, which results in lower classification accuracy. Secondly, the fact that SCAE constitutes cumulant features to the feature matrix may introduce computational redundancy. Lastly, it is much more characteristic for RNN to input signals' real parts and imaginary parts.

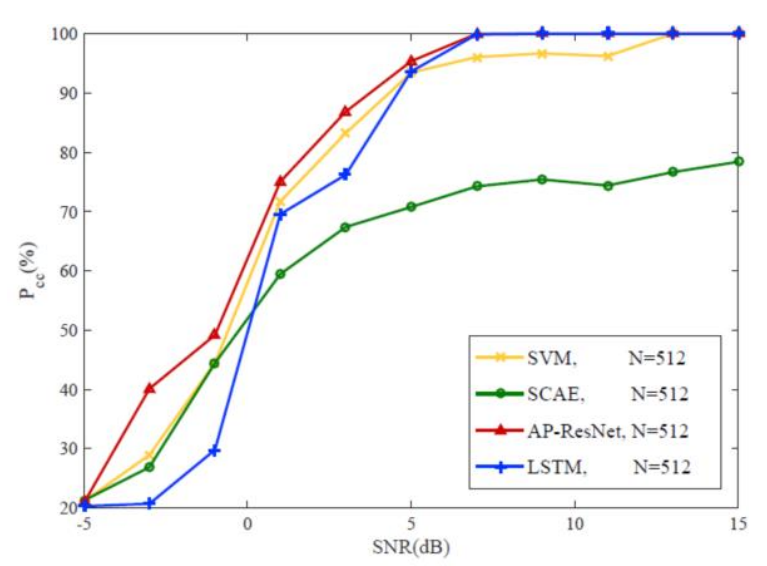

Figure 7. The classification accuracy of different methods with 512 nodes

\subsection{Classification Accuracy of Different Expand Methods}

In order to verify the effect of the sliding window method proposed in this paper, we extend the signal-to-noise ratio (SNR) of 128 points on RadioML2016.10a data set to $5 \mathrm{~dB}$. The repeated multiple replication sample method, multiple random splitting sample method, and sliding window method were selected to calculate the classification accuracy as shown in Table 2. Note that the accuracy is on the test data set.

Table 2. The classification accuracy of different expand methods

\begin{tabular}{cccc}
\multicolumn{4}{c}{ Table 2. The classification accuracy of different expand methods } \\
\hline Methods & Original & Duplicate & Multiple random split \\
\hline$P_{\mathrm{cc}}$ & $90.01 \%$ & $90.04 \%$ & $91.37 \%$ \\
\hline
\end{tabular}

Table 2 shows that the sliding window method has the highest accuracy, followed by the multiple random sample splitting method. The sliding window method has higher accuracy because it retains the internal correlation of samples, while the random split sample method has better robustness because it has been scattered several times. Compared with the original sample without expansion, the replication method only uses a single replication method to increase the number of samples, and its accuracy has almost no improvement. This also illustrates that the method of multiple replication to increase the number of samples will not improve the accuracy, but it will increase the computational complexity.

\subsection{Computation Time of Different Methods}

In order to compare the differences in computing time between the proposed method and others, we choose RadioML2016.10a to generate 256 data points on the data set. We use a Linux server for all the method and Nvidia graphics card 2 to compare the CPU computing time. The comparison is shown in Table 3. The times noted in the table are CPU time.

Table 3. Computation time of different methods

\begin{tabular}{ccccc}
\hline Methods & Our method & RNN & SCAE & SVM \\
\hline CPU computation time (seconds) & 7428.6 & 13800.3 & 15200.2 & 6128.3 \\
\hline
\end{tabular}

As can be seen from Table 3, AP-ResNet has the lowest computational complexity. Although the method of combining SVM with cumulant has the lowest computational time complexity, the accuracy of this method is poor. The reasons for the low time complexity of this method are as follows. Firstly, AP-ResNet uses CNN with ResNet structure, while the time 
complexity of CNN is lower than that of RNN. Secondly, although the computational complexity of SVM is low, it uses higher-order cumulant as input, which increases the computational complexity. Finally, the time complexity of input of SCAE application is not low. On the basis of higher-order cumulants, the feature matrix is further generated, and the SCAE network is time-consuming, so AP-ResNet has the lowest computational time complexity.

\subsection{Confusion Matrix Analysis}

Figure 8 demonstrates a series of confusion matrixes at different SNRs, and Figure 8 reveals the confusion matrixes with different symbol lengths. For each confusion matrix, the column represents the predicted category, and the row represents the real category. The colour on each grid denotes the probability. A darker grid indicates a higher probability. Taking the confusion matrix at $3 \mathrm{~dB}$ and $5 \mathrm{~dB}$ as an example, Figure 8 (e) and (f) indicate that 16QAM is easily mistaken for 64QAM. Because 16QAM constellation points are contained by 64QAM, it is difficult to identify the modulation types between 16QAM and 64QAM. However, with the increase in SNR, the accuracy increases obviously as shown in Figure 8. The influence of sample lengths is also shown in Figure 9. The SNR is fixed at 3dB. With the increase in sample nodes, the misclassification probability decreases.

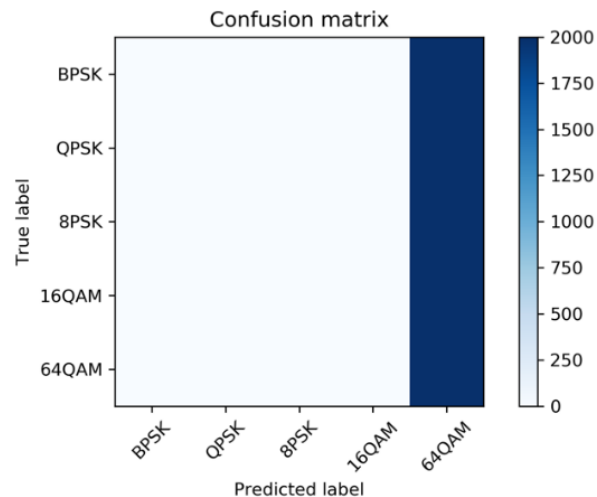

(a) $\mathrm{SNR}=-5 \mathrm{~dB}$

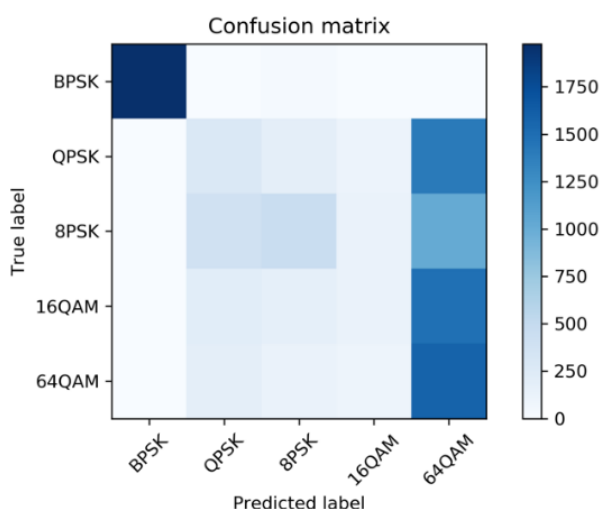

Predicted label
c) $\mathrm{SNR}=-1 \mathrm{~dB}$

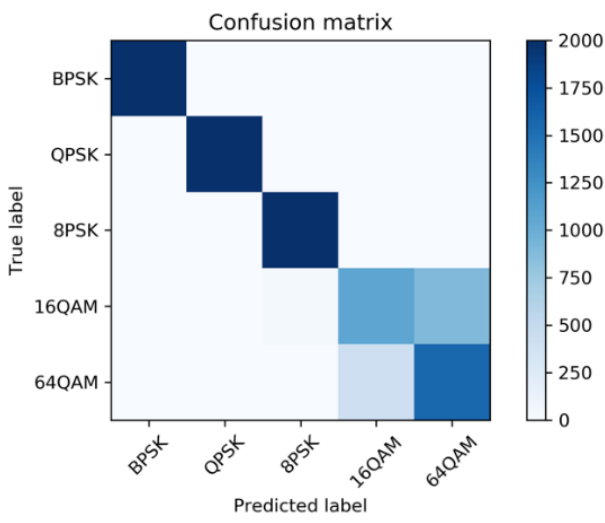

(e) $\mathrm{SNR}=3 \mathrm{~dB}$

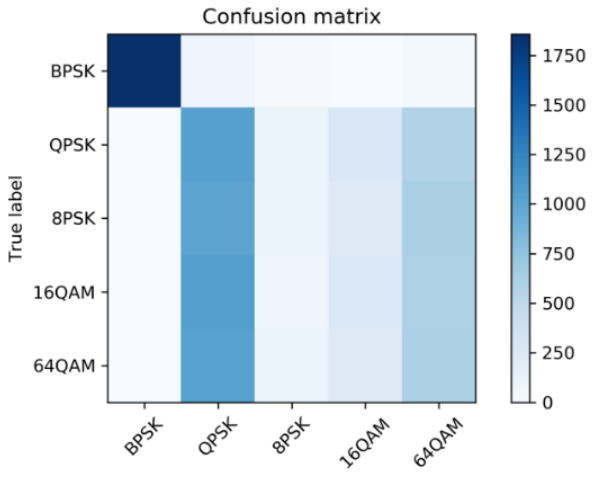

Predicted label



Predicted label
(d) $\mathrm{SNR}=1 \mathrm{~dB}$

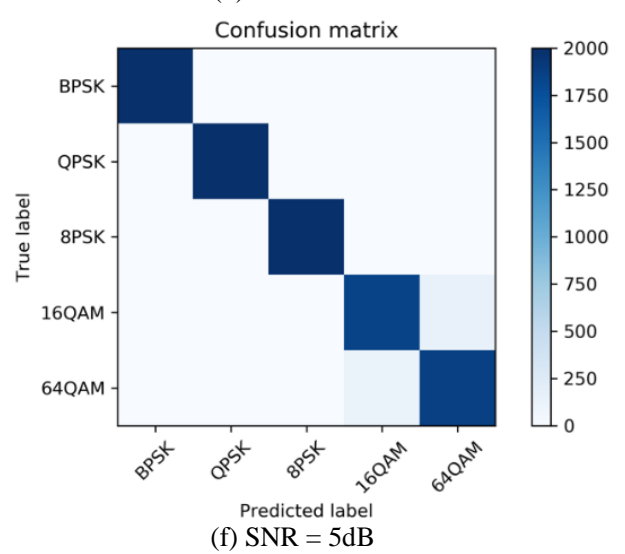

Figure 8. Confusion matrix with different SNR 




(a) Symbol length $=128$



(c) Symbol length $=512$

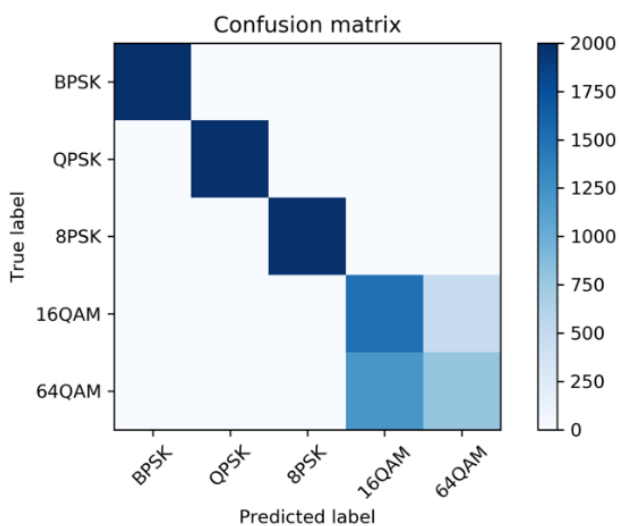

(b) Symbol length $=256$

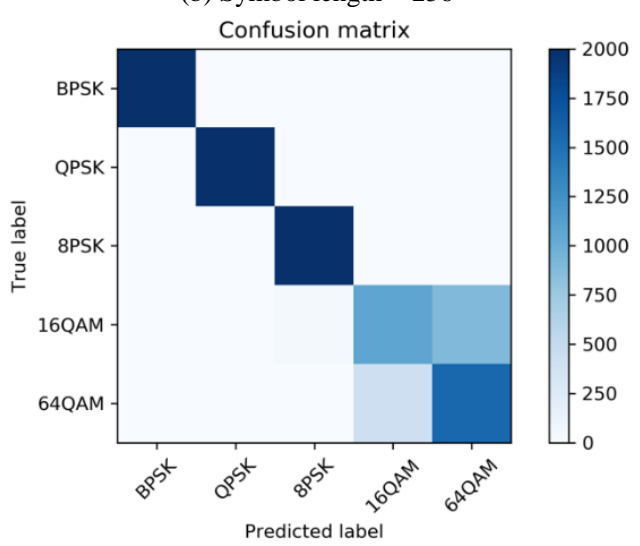

(d) Symbol length $=1024$

Figure 9. Confusion matrix with different nodes

\subsection{Robustness Performance Comparison}

Figure 10 compares the robustness of AP-ResNet, SCAE, and SVM. The frequency offset is normalized by the sample frequency. In Figure 10, it can be verified that the frequency offset degrades the classification accuracy and the AP-ResNet performs best in these scenes. Moreover, the proposed AP-ResNet achieves nearly stable classification performance, proving its robustness to frequency offset.

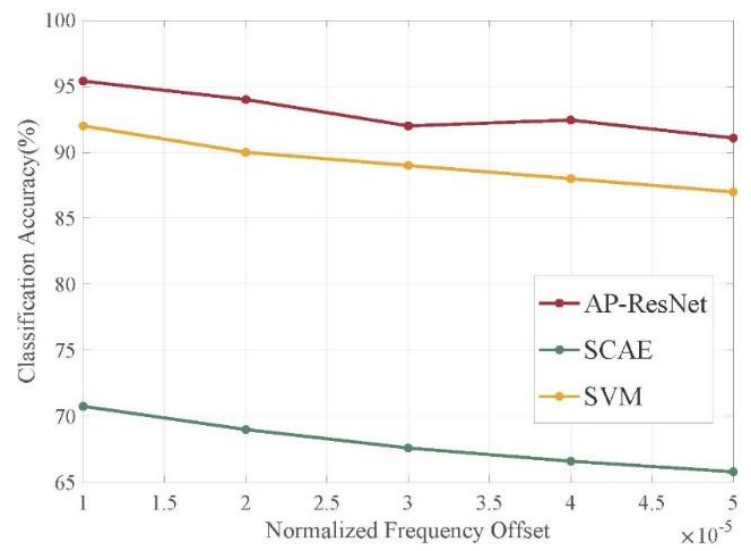

Figure 10. The robustness comparison of different methods

\section{Conclusions}

An automatic modulation recognition method named AP-ResNet, which is based on CNN (convolutional neural network) with ResNet (Residual Network) structure, is proposed in this paper. This method takes the amplitude-phase information of input signal as the original input, and a convolutional neural network with residual network structure is designed to identify 
the modulation type. Moreover, a sample expansion method based on sliding window is proposed. The proposed AP-ResNet takes advantage of the ResNet structure and combines it with the convolutional neural network to achieve superior performance. At the same time, the sliding window method can expand the sample length and preserve the internal information correlation of the sample.

\section{Acknowledgements}

This work was supported in part by the National Natural Science Foundation of China, National Key Research and Development Program of China, Key Laboratory of Dynamic Cognitive System of Electromagnetic Spectrum Space (Nanjing Univ. Aeronaut. Astronaut.), Ministry of Industry and Information Technology, and Fundamental Research Funds for the Central Universities.

\section{References}

1. Y. Lin, Y. Li, X. Yin, and Z. Dou, "Multisensor Fault Diagnosis Modeling based on the Evidence Theory," IEEE Transactions on Reliability, Vol. 67, No. 2, pp. 513-521, 2018

2. S. Huang, Y. Yao, Z. Wei, Z. Feng, and P. Zhang, "Automatic Modulation Classification of Overlapped Sources using Multiple Cumulants," IEEE Transactions on Vehicular Technology, Vol. 66, No. 7, pp. 6089-6101, 2017

3. O. A. Dobre, A. Abdi, Y. Bar-Ness, and W. Su, "Blind Modulation Classification: A Concept whose Time Has Come," IEEE Sarnoff Symp, pp. 223-228, Princeton, New Jersey, USA, April 2005

4. O. A. Dobre, "Signal Identification for Emerging Intelligent Radios: Classical Problems and New Challenge," IEEE Instrumentation and Measurement Magazine, Vol. 18, No. 2, pp. 11-18, April 2015

5. S. Huang, Y. Jiang, X. Qin, Y. Gao, Z. Feng, and P. Zhang, "Automatic Modulation Classification of Overlapped Sources using Multi-Gene Genetic Programming with Structural Risk Minimization Principle,” IEEE Access, Vol. 6, pp. 48827-48839, August 2018

6. V. D. Orlic and M. L. Dukic, "Automatic Modulation Classification Algorithm using Higher-Order Cumulants under RealWorld Channel Conditions," in Proceedings of International Conference on Telecommunication in Modern Satellite, pp. 917919, 2009

7. C. M. Spooner, "On the Utility of Sixth-Order Cyclic Cumulants for RF Signal Classification," in Proceedings of Conference Record of Thirty-Fifth Asilomar Conference on Signals, Systems and Computers, Pacific Grove, CA, USA, August 2001

8. K. Shen and E. J. Delp, "Wavelet based Rate Scalable Video Compression," IEEE Transactions on Circuits and Systems for Video Technology, Vol. 9, No. 1, pp. 109-122, February 1999

9. W. A. Gardner and C. M. Spooner, "Cyclic Spectral Analysis for Signal Detection and Modulation Recognition," in Proceedings of Military Communications Conference (MILCOM), pp. 419-424, CA, USA, October 1988

10. K. Kim, I. A. Akbar, K. K. Bae, J. S. Urn, C. M. Spooner, and J. H. Reed, "Cyclostationary Approaches to Signal Detection and Classification in Cognitive Radio," in Proceedings of IEEE International Symposium Dynamic Spectrum Access Netw. (DySPAN), pp. 212-215, Dublin, Ireland, April 2007

11. A. Swami and B. M. Sadler, "Hierarchical Digital Modulation Classification using Cumulants," IEEE Transactions on Communications, Vol. 48, No. 3, pp. 416-429, March 2000

12. Y. Tu, Y. Lin, J. Wang, and J. -U. Kim, "Semi-Supervised Learning with Generative Adversarial Networks on Digital Signal Modulation Classification," CMC-Computers Materials and Continua, Vol. 55, No. 2, pp. 243-254, 2018

13. Y. Lin, C. Wang, J. Wang, and Z. Dou, "A Novel Dynamic Spectrum Access Framework based on Reinforcement Learning for Cognitive Radio Sensor Networks," Sensors, Vol. 16, No. 10, pp. 1-22, 2016

14. Y. Lin, X. Zhu, Z. Zheng, Z. Dou, and R. Zhou, "The Individual Identification Method of Wireless Device based on Dimensionality Reduction and Machine Learning," Journal of Supercomputing, Vol. 5, pp. 1-18, 2017

15. M. Narendar, A. P. Vinod, A. S. M. Kumar, and A. K. Krishna, "Automatic Modulation Classification for Cognitive Radios using Cumulants based on Fractional Lower Order Statistics," in Proceedings of General Assembly and Scientific Symposium, Istanbul, Turkey, 2011

16. S. Huang, L. Chai, Z. Li, D. Zhang, Y. Yao, Y. Zhang, et al., "Automatic Modulation Classification using Compressive Convolutional Neural Network," IEEE Access, Vol. 1, No. 1, pp. 99, June 2019

17. S. Huang, Y. Jiang, Y. Gao, Z. Feng, and P. Zhang, "Automatic Modulation Classification using Contrastive Fully Convolutional Network," IEEE Wireless Communications Letters, Vol. 1, No. 1, pp. 99, March 2019

18. Z. Zhang, Z. Hua, and Y. Liu, "Modulation Classification in Multipath Fading Channels using Sixth-Order Cumulants and Stacked Convolutional Auto-Encoders," IET Communications, Vol. 11, No. 6, pp. 910-915, April 2017

19. S. Rajendran, W. Meert, D. Giustiniano, V. Lenders, and S. Pollin, "Deep Learning Models for Wireless Signal Classification with Distributed Low-Cost Spectrum Sensors," IEEE Transactions on Cognitive Communications and Networking, Vol. 4, No. 3, pp. 433-445, October 2018 